Running head: Attenuating Memory Distrust

\author{
Attenuating Memory Distrust in a Repeated Checking Task. \\ Mark J. Boschen*, K. Louise Wilson, and Lara J. Farrell \\ School of Psychology and Griffith Health Institute \\ Griffith University
}

Australia

*Correspondence concerning this article should be addressed to:

Dr Mark Boschen, School of Psychology, Griffith University, Southport, Australia.

Ph: +61 7 55528283, Fax: +61 7 55528291, m.boschen@griffith.edu.au 


\begin{abstract}
Repeated checking has been demonstrated to lead to reductions in memory confidence in several previous studies using student and clinical samples. This process of reduced confidence in memory and detail for memory, are thought to arise from the inhibition of perceptual processing that develops during repeated checking. Our research investigated whether reduced memory confidence from repeated checking could be attenuated through the use of novel stimuli during the repeated checking task. Three groups were generated through random assignment of 65 undergraduate students. As seen in previous research, individuals who repeatedly checked a stimulus (a virtual stovetop) showed reduced memory confidence, vividness, and detail, when compared with individuals who repeatedly checked a different stimulus. A third group in which the colour of the repeatedly-checked stovetop changed every five trials showed no significant decline in memory confidence between the pre-test and post-test. Results suggest that increased memory distrust can be ameliorated through the use of stimuli with characteristics that are novel and distinctive. Findings are discussed in the context of the existing model of repeated checking and memory confidence, and implications for treatment methods are presented.
\end{abstract}

KEYWORDS: Compulsive checking, OCD, cognitive therapy, memory confidence. 


\section{Attenuating Memory Distrust in a Repeated Checking Task.}

Despite a growing body of research into the understanding and treatment of OCD (Boschen, 2008), and the development of effective treatments for even the most severe cases (Boschen, Drummond, \& Pillay, 2008), many individuals do not experience full remission following treatment (Fisher \& Wells, 2005). Predicting those who will respond to treatment can be difficult (e.g., Boschen, Drummond, Pillay, \& Morton, 2010), and relapse is a significant problem even in successfully treated cases (e.g., Boschen, Neumann, \& Waters, 2009). Checking behaviours are the single most common compulsion in OCD, encompassing many forms such as visual checking, mental checking, reassurance seeking, and physical checking (Ball, Baer, \& Otto, 1996; Rachman, 2002; Rachman, 2003).

\section{Confidence in Memory}

An influential theory of repeated checking has suggested that individuals with checking compulsions may be motivated to recheck as a result of poor memory confidence, rather than having specific memory deficits. An experimental paradigm developed by van den Hout and Kindt (2003) presented initial evidence that repeated checking is associated with a reduction in memory confidence, while memory accuracy remains comparatively intact. In this study, the authors used a simulated 3D stovetop-checking task, where participants were instructed to turn on three out of a set of six gas burners, turn them off, and then check that they were off, over a series of 20 trials. After the 20th trial participants rated their memory confidence (i.e., whether they could accurately identify which three burners they had just used) and outcome confidence (i.e., whether the stove burners were off), along with vividness and detail of their memory. In addition, memory accuracy was also assessed by 
asking which three burners were checked in the last stovetop trial. Results revealed a paradoxical effect: Repeatedly checking a stimulus reduced memory confidence, vividness, and detail. Such consequences lead authors to suggest that repeated checking increases familiarity for the checked item, inhibiting perceptual processing and promoting conceptual processing. Conceptual processing gives heavier weighting to semantic aspects of an event, which results in the formation of a meta-memory of perceptual elements like colour and shape, thereby creating a memory that is less vivid and detailed. The authors suggested that a meta-memory is formed for all checks, reducing the individual detail and vividness of each single check, in turn leading to a reduction in memory confidence.

The meta-memory theory of compulsive checking has been replicated in several subsequent experimental studies. The checking paradigm has been replicated in real (rather than virtual) stovetops (Coles, Radomsky, \& Horng, 2006; Radomsky, Gilchrist, \& Dussault, 2006), mental (rather than physical) checking (Radomsky \& Alcolado, 2010), and also in clinical (rather than student) samples of individuals with OCD (Boschen \& Vuksanovic, 2007).

\section{Attenuating Reduction in Memory Confidence}

Despite several studies which have examined the process by which repeated checking leads to memory distrust, there has been little research into mechanisms by which this effect may be attenuated. Such research may yield important clues regarding methods that could be incorporated into current treatments of compulsive checking. A key component of van den Hout \& Kindt's (2003) model is that the act of repeated checking leads to a shift from perceptual to conceptual processing, followed by a reduction in memory vividness and detail, which in turn leads to a fall 
in memory confidence. Two previous studies have examined different methods of enhancing memory vividness and detail to reduce doubt in repeated checking.

An early study examining the effect of increasing novelty and disctinctinvenes was conducted by Tallis in 1993. Three participants with OCD engaged in a doubt reduction strategy involving the deliberate pairing of successful actions (e.g., successfully turning off an iron) with a distinct, novel stimulus (e.g., a cardboard blue square). Tallis suggested that distinctive stimuli would have a 'stand out' effect, thus aiding recall, and confidence in the memory. When participants then felt the urge to check, they visualised the shape they associated with the last successful completion of the task. This was repeated (with a different colour and size shape) for each time they checked the same stimulus. Repeated checking behaviours were reduced in all three cases. While these results appear promising, the small number of participants, and lack of a control condition suggest the need to replicate findings with larger samples and controlled experimental methodology.

A more direct attempt to manipulate memory confidence using a method similar to that of van den Hout and Kindt (2003) was conducted using a real stovetop task by a study by Ashbaugh and Radomsky (2007). In this study, the authors attempted to shift a participant's attentional focus in order to attenuate reductions in memory confidence. The study aimed to increase perceptual features of a check by shifting the focus from solely threat-relevant stimuli to both threat-relevant and threat-irrelevant stimuli. Participants were instructed to attend to the stimulus (real stovetop) and also surrounding peripheral details of the checking environment (other parts and objects within the kitchenette). A control group focused their attention solely on the stovetop. The aim was to increase awareness of the contextual environment in which checking takes place, in order to make the memory for 
adequately checking a stovetop more vivid and distinct. The findings, however, revealed no differences between peripheral and central focused groups, including no differences in memory confidence. Notably, memory accuracy was superior in the peripherally focused group compared to the central focus group. Unfortunately, this study (Ashbaugh \& Radomsky, 2007) did not include a no attention control group, thus the full experimental effects of peripheral focus of attention on memory and meta-memory theories of compulsive checking cannot be ascertained from their results. Furthermore, this study did not actively enhance the distinctiveness of each check, and therefore may not have sufficiently inhibited the shift from perceptual to conceptual processing.

\section{The Present Study}

The present study examined the effects of novelty and distinctiveness on memory confidence and related variables in a repeated checking paradigm. Student participants were randomly assigned to either (a) an original stovetop condition where individuals would repeatedly check stovetops; (b) a lights control condition where participants would repeatedly check an array of lights; or (c) a perceptual change condition, where individuals would repeatedly check a stovetop that changed in colour over the trials in an attempt to increase the distinctiveness of different trials.

A number of a priori hypotheses were formulated, each based on previous findings in the literature. We aimed to experimentally manipulate the distinctiveness and novelty of a stimulus, leading to a reduced shift from perceptual to conceptual processing. It was hypothesised that this would reduce the formation of a metamemory as a result of repeated checking, which would in turn attenuate reductions in memory confidence. 
Specifically, it was hypothesised that (a) there would be significant group differences across trials on memory confidence, whereby there would be significant decline in memory confidence for the original stovetop condition compared to the control condition; and that there would be significantly less decline in memory confidence for the perceptual change condition compared to the original stovetop condition. Furthermore, (b) it was predicted the same pattern of group differences would be observed for related variables of memory vividness and memory detail. Finally, it was hypothesised that (c) urge to check would also be significantly reduced for the perceptual change condition, compared to the original stovetop condition, with urge to check being the product of reduced memory confidence. Other variables such as outcome confidence and memory accuracy were not expected 
to show declines from repeated checking, or differences between experimental conditions.

\section{Method}

\section{Participants}

The sample consisted of 65 first-year psychology students at an Australian university who received course credit in exchange for their participation. Participants were randomly assigned across the different conditions. The sample comprised 57 $(87.7 \%)$ females, and $8(12.3 \%)$ males. The mean age was 24.18 years $(S D=7.48)$. All participants had normal, or corrected-to-normal vision. Two (3.1\%) participants 
were receiving treatment for OCD at the time of the experiment. ${ }^{1}$ Other previous anxiety disorder treatment was reported by 10 (15.4\%) participants.

Materials

3D Checking Task (van den Hout \& Kindt, 2003). This task was used to determine the effects of repeated checking on an individual's memory confidence. The task 7 participants to manipulate a 3D virtual stovetop and series of light bulbs, as outlined in the Procedure section below. The virtual stovetop was modified from the original software used in van den Hout \& Kindt (2003).

In each trial of the 3D Checking Task, participants were required to complete three different steps, involving the manipulation of an array of either six gas stove

\footnotetext{
${ }^{1}$ All tests of significance were rerun with these two participants excluded. In all cases the significance of the results was unchanged.
} 
burners, or six incandescent lights. In each trial they were asked to turn on a randomly generated subset of three of the six lights/burners using an on-screen dial (burners) or slider (lights). They were then asked to turn off these three lights/burners. Finally, the participant was asked to check that each of the three lights/burners was off, by manipulating the dial/slider. All participants completed 20 trials in between a pre-test and post-test trial in which all participants used the stovetop. A detailed description of the 3D Checking task has been described previously by van den Hout \& Kindt (2003).

Checking Task Questionnaire (van den Hout \& Kindt, 2003). The Checking Task Questionnaire was administered to participants after pre-test and post-test on the 3D stovetop. The questionnaire required participants to mark which three of the six burners were manipulated in the last checking episode, as a measure of memory accuracy. Participants were given a score calculated according the following formula: $3 \times$ (number of correctly selected burners $\div$ total number of selected burners), to adjust for participants who selected more than three burners. Following this question, participants were asked to rate memory vividness, memory detail, memory confidence, outcome confidence, and urge to check, using a scale from 1 (indicative of a low score) to 10 (indicative of a high score). For example, for memory confidence, the item was "how confident are you that you turned off all three burners?”

Obsessive-Compulsive Inventory (OCI; Foa et al., 1998). The OCI is a 42item self-report questionnaire that measures frequency and distress of OCD symptoms, with subscales for washing, checking, doubting, ordering, obsessing, hoarding, and mental neutralizing. The OCI differentiates between OCD, other anxiety disorders, and non-clinical participants and shows good internal consistency 
and test-retest reliability. We employed this measure to quantify the level of OCD symptoms in the sample group. Cronbach's $\alpha$ for the current sample was calculated for the overall OCI frequency $(\alpha=.94)$ and distress scales $(\alpha=.95)$, and additionally for frequency ( $\alpha=.87)$ and distress $(\alpha=.87)$ scores for the checking subscale. This is similar to reliability statistics reported by Foa et al. in their non-clinical sample, which reported a Cronbach’s a of .90 and .89 for overall frequency and distress scales, and .80 for the frequency and distress checking subscales.

Procedure

The experiment took place in a dimly lit, sound-attenuated room, over approximately 45 minutes. Standardised instructions were given verbally and on the computer screen to the participants. Participants completed the checking trials under either a Lights Control (LC) condition, Original Stovetop (OS) condition, or Perceptual Change (PC) condition. All participants then completed the Checking Task Questionnaire followed by the OCI, and a demographics questionnaire.

Lights Control (LC) Condition. The LC condition was procedurally identical to the original van den Hout \& Kindt (2003) control condition. Participants completed a single pre-test trial of the stovetop task, along with the Checking Task Questionnaire. This was followed by 20 trials in which they manipulated and checked the array of six lights, and then finally a single trial using the stovetop as a post-test.

Original Stovetop (OS) Condition. Participants assigned to the OC condition followed the same procedure as the experimental group in the original van den Hout \& Kindt (2003) experiment. This was identical to the LC condition, except each participant completed the 20 trials using the stovetop. 
Perceptual Change (PC) Condition. In the PC condition, participants performed the checking task similarly to the OS condition, with an added element of colour manipulation. The 20 stovetop checking trials were broken into blocks of five, with the colour of the burners and an additional star on each burner changed across each block. The colours of the burners (in order) were green, blue, purple and black, while the figure of a star was coloured red, orange, yellow, and then white.

\section{Results}

Data from three participants were removed from the dataset due to unusual scores on one or more variables. Two of these participants (from the SC condition) endorsed all items at maximum for the entire questionnaire set. One of these participants (from the PC condition) endorsed all items except one at the minimum score for the entire question set.

\section{Equivalence of Experimental Conditions}

The three experimental conditions were equivalent in overall OCD symptom frequency and distress, and compulsive checking symptom frequency and distress. At the pre-test, memory accuracy, memory vividness, memory detail, memory confidence, outcome confidence, and urge to check were all equivalent across conditions. All groups regarded the difficulty of the task as similar. Table 1 presents details of the baseline scores and statistical tests of equivalence.

\section{Memory Accuracy}

To examine the differences in memory accuracy after repeated checking, a 3 (Condition: LC, OS, PC groups) $\times 2$ (Time: Pre-test, Post-test) mixed factorial ANOVA was employed, with memory accuracy (i.e., number of correctly remembered stovetop burners) as the dependent variable. There was no significant 
interaction between condition and time on memory accuracy $\left(F_{(2,59)}=0.25, p=.78\right.$, partial $\eta^{2}=0.01$ ), demonstrating that memory accuracy did not differ between each condition across time. The main effect of condition was not significant $\left(F_{(2,59)}=0.72\right.$, $p=.49$, partial $\eta^{2}=0.02$ ), showing that memory accuracy did not differ among the three conditions. Similarly, the main effect of time was also not significant $\left(F_{(1,59)}=\right.$ 2.46, $p=.12$, partial $\eta^{2}=0.04$ ), demonstrating no change in memory accuracy from pre-test to post-test. Means and standard deviations from the groups are shown in Table 2.

Memory Confidence

There was a significant condition $\times$ time interaction when memory confidence was used as the dependent variable $\left(F_{(2,59)}=5.76, p=.005\right.$, partial $\left.\eta^{2}=0.16\right)$. This showed that the three conditions differed across time on the memory confidence variable (see Figure 1). There were no significant main effects either for condition $\left(F_{(2,59)}=0.55, p=.58\right.$, partial $\left.\eta^{2}=0.02\right)$, or time $\left(F_{(2,59)}=0.24, p=.62\right.$, partial $\eta^{2}=$ 0.004). Figure 1 depicts the interaction between time and condition.

The significant interaction was broken down into two simple effects analyses. As expected, participants in the OS condition reported significant reduction in memory confidence from pre-test to post-test, $\left.F_{(1,19)}=5.29, p=.033\right)$. Participants from the LC condition improved in memory confidence from pre-test to post-test $\left(F_{(1,21)}=6.59, p=.018\right)$, while those in the PC condition showed no significant change in memory confidence over the twenty trials $\left(F_{(1,19)}=0.90, p=.354\right)$.

The simple effect of condition at post-test was examined to assess for differences in memory confidence after the repeated checking task. An ANOVA revealed the expected group differences, but with borderline statistical significance $\left(F_{(2,59)}=3.16, p=.050\right.$, partial $\left.\eta^{2}=0.97\right)$. Planned pairwise comparisons revealed 
significant group differences at post-test between the LC and OS conditions (Mean Diff $=1.91, p=.015)$. There were no significant differences between other pairs. Vividness and Detail in Memory

Two separate 3 (condition) $\times 2$ (time) mixed factorial ANOVAs with repeated measures were conducted to determine whether the conditions differed in vividness and detail of memory after repeated checking. For memory vividness, the expected Condition $\times$ Time interaction was non-significant $\left(F_{(2,59)}=2.63, p=.08\right.$, partial $\eta^{2}=$ 0.082). There was no significant main effect of condition $\left(F_{(2,59)}=1.29, p=.283\right.$, partial $\eta^{2}=0.042$ ). The main effect of time demonstrated significant reduction in memory vividness from pre-test to post-test $\left(F_{(1,59)}=4.51, p=.038\right.$, partial $\eta^{2}=$ 0.71) across the three conditions. Memory detail showed no significant condition $\times$ time interaction $\left(F_{(2,59)}=1.43, p=.247\right.$, partial $\left.\eta^{2=} 0.04\right)$. Furthermore, there were no significant main effects of either time $\left(F_{(1,59)}=3.25, p=.077\right.$, partial $\left.\eta^{2}=0.05\right)$, or condition $\left(F_{(2,59)}=0.25, p=.784\right.$, partial $\left.\eta^{2=} 0.008\right)$.

\section{Outcome Confidence}

To assess for differences in outcome confidence between conditions across time, a further 3 (condition) $\times 2$ (time) mixed factorial ANOVA with repeated measures was conducted. The condition $\times$ time interaction was non-significant $\left(F_{(2,}\right.$ 59) $=0.18, p=.834$, partial $\left.\eta^{2}=0.006\right)$. The main effects for condition $\left(F_{(2,59)}=0.71\right.$, $p=.496$, partial $\left.\eta^{2}=0.023\right)$ and time $\left(F_{(1,59)}=2.13, p=.150\right.$, partial $\left.\eta^{2}=0.035\right)$ were also non-significant.

\section{Urge to Check}

Differences in urge to check between conditions and across time were assessed using a 3 (condition) $\times 2$ (time) mixed factorial AVOVA with repeated measures. The condition $\times$ time interaction was non-significant $\left(F_{(2,59)}=0.25, p=\right.$ 
.784, partial $\left.\eta^{2}=0.008\right)$, as were the main effects of condition $\left(F_{(2,59)}=0.26, p=\right.$ .773 , partial $\left.\eta^{2}=0.009\right)$, and time $\left(F_{(1,59)}=2.18, p=.146\right.$, partial $\eta^{2}=0.036$.

\section{Regression of Vividness and Detail on Memory Confidence}

To determine whether vividness and detail contribute significantly to memory confidence after repeatedly checking, and to determine whether, the change in vividness and detail from pre to post-test contributed uniquely to the change in memory confidence from pre to post-test, two multiple regressions were run. As the literature on the repeated checking paradigm assumes both vividness and detail contribute equally to an individual's confidence for last checked stovetop, a multiple regression was considered more suitable than a hierarchical regression. For the first multiple regression, post-test memory confidence was used as the dependent variable, with post-test vividness and detail as the predictors. Vividness and detail accounted for $57.6 \%\left(R^{2}=.576, F_{(2,59)}=40.14, p<.001\right)$ of the variance in confidence in memory. Only post-test memory detail made a significant contribution to the variance in post-test memory confidence, as shown in Table 3. The strong correlation $(r=.81, p<.001)$ between memory vividness and memory detail, however, is likely to have led to multi-colinearity which may account for the nonsignificant additional contribution of memory vividness.

For the second multiple regression, change in confidence was used as the dependent variable, with change in vividness and detail as the predictors. Degree of change statistics were calculated by subtracting the pre-test score from the post-test score for vividness, detail and memory confidence variables. A positive score meant that there was a decline in memory confidence from pre to post-test trials. When the change in vividness and detail were used as a predictor of change in memory confidence $43.7 \%$ of the variance was accounted $\left(R^{2}=0.437, F_{(2,79)}=22.91, p<\right.$. 
001. Only change in memory detail made a significant unique contribution to a change in memory confidence. This was likely to be the result of multi-colinearity with bivariate correlations between these two variables of $r=.83(p<.001)$. The multiple regression statistics for each of the predictors are presented in Table 3.

\section{Discussion}

This study investigated the ability of novel and distinctive stimuli to attenuate the reductions in memory confidence usually observed as the result of repeated checking. Our study confirmed earlier findings (e.g., Boschen \& Vuksanovic, 2007; Coles et al., 2006; Radomsky et al., 2006; van den Hout \& Kindt, 2003) that repeated checking of a stovetop is associated with reductions in memory confidence, relative to a control condition in which a different object was checked. This occurred despite no significant changes in memory accuracy. The LC condition, in which participants repeatedly checked lights, experienced an increase in memory confidence from the pre-test to the post-test. When the colours of the stimulus were changed every five checking trials (i.e., in the PC condition), this attenuated the reductions in memory confidence, with no significant change from pre-test to post-test. At the post-test, participants in this PC condition reported memory confidence levels that were not significantly different from the LC control group, but also not significantly better than the group where the stovetop colours were held constant. Despite the fact that memory vividness and memory detail did not show the same findings in the comparisons of group means, regression provided support for the relationship between these variables and memory confidence.

Integration with Previous Theory 
According to van den Hout and Kindt (2003), when an object is checked repeatedly this results in the formation of a memory amalgam in which the specific perceptual details of each check are lost. In the consolidation of this memory perceptual processing is inhibited in favour of conceptual processing, which is associated with less vividness and detail when the individual attempts to recall individual checking memories is recalled. Reductions in memory vividness and detail promote distrust in the recalled memory.

This theory of repeated checking would suggest that methods which enhance the distinctiveness of individual memories may be able to reduce the consolidation of these memories into a more general meta-memory. Our experiment demonstrated that by manipulating the novelty and distinctiveness of repeatedly checked stimuli, this led to less reduction in memory confidence over 20 checking trials. Novelty and distinctiveness have been shown previously to increase perceptual processing (Hirshman \& Mulligar, 1991), suggesting that by making the stovetop stimuli novel and distinctive, this may have enhanced perceptual processing, increasing the vividness and detail of the post-test checking trial. In our study, reductions in memory confidence were related to changes in memory vividness/detail, supporting this model of repeated checking. Implications, Limitations, and Future Directions

We believe that this study has several implications that are important for the understanding of repeated checking behaviour, the occurrence of this behaviour in clinical conditions such as OCD, as well as potential methods that might be used to reduce this behaviour. Firstly, the current study contributes to a growing understanding of the self-defeating effects of repeated checking on memory confidence. It confirms earlier findings that repeated checking leads to decreased 
confidence in memory for recent checking episodes, and that this is related to reductions in memory vividness and detail. Importantly, repeated checking leads to reduced memory confidence, rather than reduced memory accuracy, suggesting that repeated checking arises from low confidence not accuracy. Secondly, the current study adds to previous suggestions of a mechanism that may contribute to repeated checking seen in conditions such as OCD. If individuals with OCD check to feel more confident in the success of their behaviour, this may paradoxically reduce the confidence in their memory, increasing their perceived need to recheck.

A third implication of our study arises from our observation that the effect of repeated checking on memory confidence can be reduced. The use of distinctive stimuli to reduce the memory distrust from repeated checking is the first demonstration of the ability to mitigate these effects. If reduced memory confidence can be modified in experimental contexts such as ours, this provides suggestions that related methods may assist in the treatment of repeated checking. We are aware of only one study that has previously attempted to use stimulus novelty as a mechanism to reduce compulsive checking in OCD. Tallis (1993)'s successful attempt to reduce checking in three individuals with OCD involved the use of external aids to enhance the distinctiveness and novelty of each individual check. Our results are in accordance with these earlier findings.

The reader should remain mindful of the limitations in this study in interpreting the findings presented here. Firstly, as this was the first study to demonstrate a reduction in memory distrust, the findings require replication. Secondly, our results are based on findings observed in a non-clinical sample of undergraduate students. It remains to be demonstrated that the reductions in memory distrust from repeated checking would be also observed in a group of individuals with 
compulsive checking problems. To date only one study (Boschen \& Vuksanovic, 2007) has utilised clinical participants using the repeated checking paradigm of van den Hout and Kindt (2003). In this study, the same effects of repeated checking were observed in clinical and non-clinical samples. Some differences were noted, however: that there was a greater impact of repeated checking on memory confidence, and that increased perceived responsibility had a further effect in clinical participants. These differences suggest that while the basic process of repeated checking reduces memory confidence across both clinical and non-clinical samples, there are also differences in the underlying mechanisms of how these processes operate. These differences highlight the need for replication with clinical samples.

Another limitation from our study concerns the generalizability of the method used here to one which may be useful to reduce compulsive checking. Our method relied on automatic changes to the checked stimulus that increased the novelty of the stimulus over the repeated checking trials. Such automatic changes in the checked stimulus are different to the usual appearance of items that are checked repeatedly in OCD. For example, a person who compulsively rechecks that a stove is off before leaving the house is exposed to a checking stimulus (the stovetop) which is essentially the same for each check. In this case, changes to the appearance of the stovetop are difficult to implement as a means of enhancing perceptual distinctiveness. Future research may investigate methods similar to that of Tallis (1993) in using more 'portable' methods of increasing the distinctiveness of the rechecked object, rather than automatic changes in the items themselves.

The utility of stimulus novelty over the longer-term is not addressed by the current research. Each participant in our study was involved in the task for less than one hour, and was not required to use any more than 4 distinctive stimuli over 20 
checking trials. It is possible that the benefits of stimulus novelty may fade over time. This would be consistent with the theory proposed by van den Hout \& Kindt (2003) in that the stimuli that were initially novel would become less so, and as a result would lose their ability to increase memory vividness, detail, and confidence. In Tallis' (1993) three individuals who used distinctive stimuli to reduce compulsive checking, there was no data available for follow-up beyond one year. As such the longer term effectiveness of the use of distinctive stimuli remains unclear.

Our study made a total of 4 changes to the colours of the stovetop burners over the course of the 20 checking trials. Our research does not provide any information about the limits to this method in enhancing perceptual distinctiveness. There are likely to be inherent limits to how many changes in the checked stimulus can be used to maintain perceptual distinctiveness. Future research may explore the boundaries of our method over longer numbers of trials, and different numbers of changes in the rechecked stimuli.

\section{Conclusion}

Our study provides further replication of previous work which has demonstrated that the act of repeated checking leads to reduced memory confidence. In addition, it provides some of the first evidence that these reductions in memory confidence can be attenuated through the use of methods which increase the novelty and distinctiveness of the checked stimuli. Such methods may provide useful insights into techniques that may be useful in individuals with clinical compulsive checking behaviours. 


\section{References}

Ashbaugh, A.R., \& Radomsky, A.S. (2007). Attentional focus during repeated checking influences memory but not meta-memory. Cognitive Therapy and Research, 31, 291-306.

Ball, S. G., Baer, L., \& Otto, M.W. (1996). Symptom subtypes of obsessivecompulsive disorder in behavioural treatment studies: A quantitative review. Behaviour Research and Therapy, 34, 47-51.

Boschen, M. J. (2008). Publication trends in individual anxiety disorders: 19802015. Journal of Anxiety Disorders, 22, 570-575.

Boschen, M.J., Drummond, L.M., \& Pillay, A. (2008). Treatment of severe, treatment-refractory obsessive-compulsive disorder: A study of inpatient and community treatment. CNS Spectrums, 13, 1056-1065.

Boschen, M.J., Drummond, L.M., Pillay, A., \& Morton, K. (2010). Predicting outcome of treatment for severe, treatment resistant OCD in inpatient and community settings. Journal of Behavior Therapy and Experimental Psychiatry, 41, 90-95.

Boschen, M.J., Neumann, D.L., \& Waters, A.M. (2009). Relapse of successfully treated anxiety and fear: Theoretical issues and recommendations for clinical practice. Australian and New Zealand Journal of Psychiatry, 43, 89-100.

Boschen, M.J., \& Vuksanovic, D. (2007). Deteriorating memory confidence, responsibility perceptions and repeated checking: Comparisons in OCD and control samples. Behaviour Research and Therapy, 45, 2098-2109.

Coles, M.E., Radomsky, A.S., \& Horng, B. (2006). Exploring the boundaries of memory distrust from repeated checking: Increasing external validity and examining thresholds. Behaviour Research and Therapy, 44, 995-1006. 
Farrell, L.J. \& Boschen, M.J. (2010). Treatment outcome in adult OCD: Predictors and processes of change. Asia Pacific Journal of Counselling and Psychotherapy, 2, 82-97.

Foa, E. B., Kozak, M. J., Salkovskis, P. M., Coles, M. E., \& Amir, N. (1998). The validation of a new obsessive-compulsive disorder scale: The ObsessiveCompulsive Inventory. Psychological Assessment, 10, 206-214.

Fowle, H.J. \& Boschen, M.J. (2011). The impact of compulsive cleaning on confidence in memory and cleanliness. Journal of Anxiety Disorders, 25, 237243.

Fisher, P. L., \& Wells, A. (2005). How effective are cognitive and behavioural treatments for obsessive-compulsive disorder? A clinical significance analysis. Behaviour Research and Therapy, 43, 1543-1558.

Rachman, S. J. (2002). A cognitive theory of compulsive checking. Behaviour Research and Therapy, 40, 625-639.

Radomsky, A.S. \& Alcolado, G.M. (2010). Don’t even think about checking: Mental checking causes memory distrust. Journal of Behavior Therapy and Experimental Psychiatry, 41, 345-351.

Radomsky, A.S., Gilchrist, P.T., Dussault, D. (2006). Repeated checking really does cause memory distrust. Behaviour Research and Therapy, 44, 305-316.

Rachman, S. J. (2003). Compulsive checking. In: R. G. Menzies \& P. de Silva (Eds.), Obsessive-compulsive disorder: theory, research and treatment (pp. 138-180). West Sussex: Wiley.

Tallis, F. (1993). Doubt reduction using distinctive stimuli as a treatment for compulsive checking: An exploratory investigation. Clinical Psychology and Psychotherapy, 1, 45-52. 
van den Hout, M. A, \& Kindt, M. (2003). Repeated checking causes memory distrust. Behaviour Research and Therapy, 41, 301-316. 


\section{Author Note}

This research was conducted as part of the Bachelor of Psychology (Honours) dissertation by the second author, under the supervision of the first author.

We wish to thank Professor Marcel van den Hout for permission to use the 3D Stovetop software, and invaluable advice on the development of the application. 
Table 1

Baseline Measurements and Tests of Equivalence

\begin{tabular}{|c|c|c|c|c|c|c|c|}
\hline \multirow[t]{2}{*}{ Variable } & \multicolumn{2}{|c|}{ Lights Control Group $(n=22)$} & \multicolumn{2}{|c|}{ Original Stovetop Group $(n=20)$} & \multicolumn{2}{|c|}{$\begin{array}{l}\text { Perceptual Change Group ( } n= \\
\text { 20) }\end{array}$} & \multirow[t]{2}{*}{ Significance Test } \\
\hline & $M$ & $S D$ & $M$ & $S D$ & $M$ & $S D$ & \\
\hline OCI Total Frequency & 41.91 & 29.00 & 37.60 & 27.50 & 38.85 & 21.26 & $F_{(2,59)}=0.15, p=.86$ \\
\hline OCI Total Distress & 31.23 & 21.98 & 32.30 & 28.31 & 24.85 & 21.15 & $F_{(2,59)}=0.57, p=.57$ \\
\hline OCI Checking Frequency & 1.08 & 0.86 & 0.98 & 0.87 & 0.96 & 0.61 & $F_{(2,59)}=0.14, p=.87$ \\
\hline OCI Checking Distress & 0.66 & 0.73 & 0.70 & 0.68 & 0.56 & 0.58 & $F_{(2,59)}=0.27, p=.77$ \\
\hline Pre-Test Memory Accuracy & 2.45 & 0.65 & 2.33 & 0.89 & 2.46 & 0.80 & $F_{(2,59)}=0.20, p=.82$ \\
\hline Pre-Test Memory Vividness & 6.86 & 2.51 & 7.00 & 2.88 & 7.85 & 1.87 & $F_{(2,59)}=0.97, p=.39$ \\
\hline Pre-Test Memory Detail & 7.00 & 2.39 & 7.30 & 2.64 & 7.05 & 2.06 & $F_{(2,59)}=0.09, p=.91$ \\
\hline Pre-Test Memory Confidence & 5.86 & 2.98 & 6.90 & 2.65 & 7.25 & 2.53 & $F_{(2,59)}=1.47, p=.24$ \\
\hline Pre-Test Outcome Confidence & 6.41 & 2.74 & 6.80 & 2.44 & 6.15 & 2.66 & $F_{(2,59)}=0.31, p=.73$ \\
\hline Pre-Test Urge to Check & 6.45 & 2.50 & 6.95 & 2.80 & 6.20 & 2.24 & $F_{(2,59)}=0.46, p=.64$ \\
\hline Subjective Task Difficulty & 4.27 & 2.35 & 4.05 & 2.06 & 4.60 & 1.98 & $F_{(2,59)}=0.33, p=.72$ \\
\hline
\end{tabular}

Note. OCI = Obsessive Compulsive Inventory 
Table 2

\section{Group Means and Standard Deviations}

\begin{tabular}{|c|c|c|c|c|c|c|c|c|c|}
\hline & \multirow[t]{2}{*}{ Timepoint } & \multicolumn{2}{|c|}{$\begin{array}{l}\text { Lights Control Group }(n= \\
22)\end{array}$} & \multicolumn{2}{|c|}{$\begin{array}{l}\text { Original Stovetop Group }(n= \\
20)\end{array}$} & \multicolumn{2}{|c|}{$\begin{array}{l}\text { Perceptual Change Group ( } n \\
=20)\end{array}$} & \multicolumn{2}{|c|}{ Total Sample $(N=62)$} \\
\hline & & $M$ & $S D$ & $M$ & $S D$ & $M$ & $S D$ & $M$ & $S D$ \\
\hline \multirow[t]{2}{*}{ Memory Accuracy } & Pre-Test & 2.45 & 0.65 & 2.33 & 0.89 & 2.46 & 0.80 & 2.42 & 0.77 \\
\hline & Post-Test & 2.57 & 0.68 & 2.45 & 0.76 & 2.75 & 0.53 & 2.59 & 0.66 \\
\hline \multirow[t]{2}{*}{ Memory Confidence } & Pre-Test & 5.86 & 2.98 & 6.90 & 2.65 & 7.25 & 2.53 & 6.65 & 2.76 \\
\hline & Post-Test & 7.41 & 2.40 & 5.50 & 2.54 & 6.55 & 2.44 & 6.52 & 2.55 \\
\hline \multirow[t]{2}{*}{ Memory Vividness } & Pre-Test & 6.86 & 2.51 & 7.00 & 2.88 & 7.85 & 1.87 & 7.23 & 2.46 \\
\hline & Post-Test & 7.18 & 2.20 & 5.70 & 2.62 & 6.85 & 1.84 & 6.60 & 2.29 \\
\hline \multirow[t]{2}{*}{ Memory Detail } & Pre-Test & 7.00 & 2.39 & 7.30 & 2.64 & 7.05 & 2.06 & 7.11 & 2.34 \\
\hline & Post-Test & 7.05 & 2.38 & 5.90 & 2.63 & 6.50 & 2.31 & 6.50 & 2.45 \\
\hline \multirow[t]{2}{*}{ Outcome Confidence } & Pre-Test & 6.41 & 2.74 & 6.80 & 2.44 & 6.15 & 2.66 & 6.45 & 2.59 \\
\hline & Post-Test & 6.73 & 2.95 & 7.55 & 2.35 & 6.50 & 2.71 & 6.92 & 2.68 \\
\hline \multirow[t]{2}{*}{ Urge to Check } & Pre-Test & 6.45 & 2.50 & 6.95 & 2.80 & 6.20 & 2.24 & 6.53 & 2.50 \\
\hline & Post-Test & 6.14 & 2.51 & 6.00 & 3.46 & 5.75 & 2.57 & 5.97 & 2.83 \\
\hline
\end{tabular}


Table 3

\section{Regression Statistics}

\begin{tabular}{lccccc}
\hline Variable & $B$ & Std Err & $\beta$ & $t$ & $p$ \\
\hline Dependent: Memory Confidence (Post-Test) & & & & & \\
Memory Vividness (Post-Test) & 0.19 & 0.16 & 0.17 & 1.21 & .231 \\
Memory Detail (Post-Test) & 0.64 & 0.15 & 0.61 & 4.26 & $<.001$ \\
\hline Dependent: Memory Confidence Change & & & & & \\
Memory Vividness Change & 0.25 & 0.22 & .20 & 1.14 & .258 \\
Memory Vividness Change & 0.55 & 0.20 & .49 & 2.79 & .007 \\
\end{tabular}




\section{Figure Captions}

Figure 1. Change in Memory Confidence over Time for the Three Conditions. 


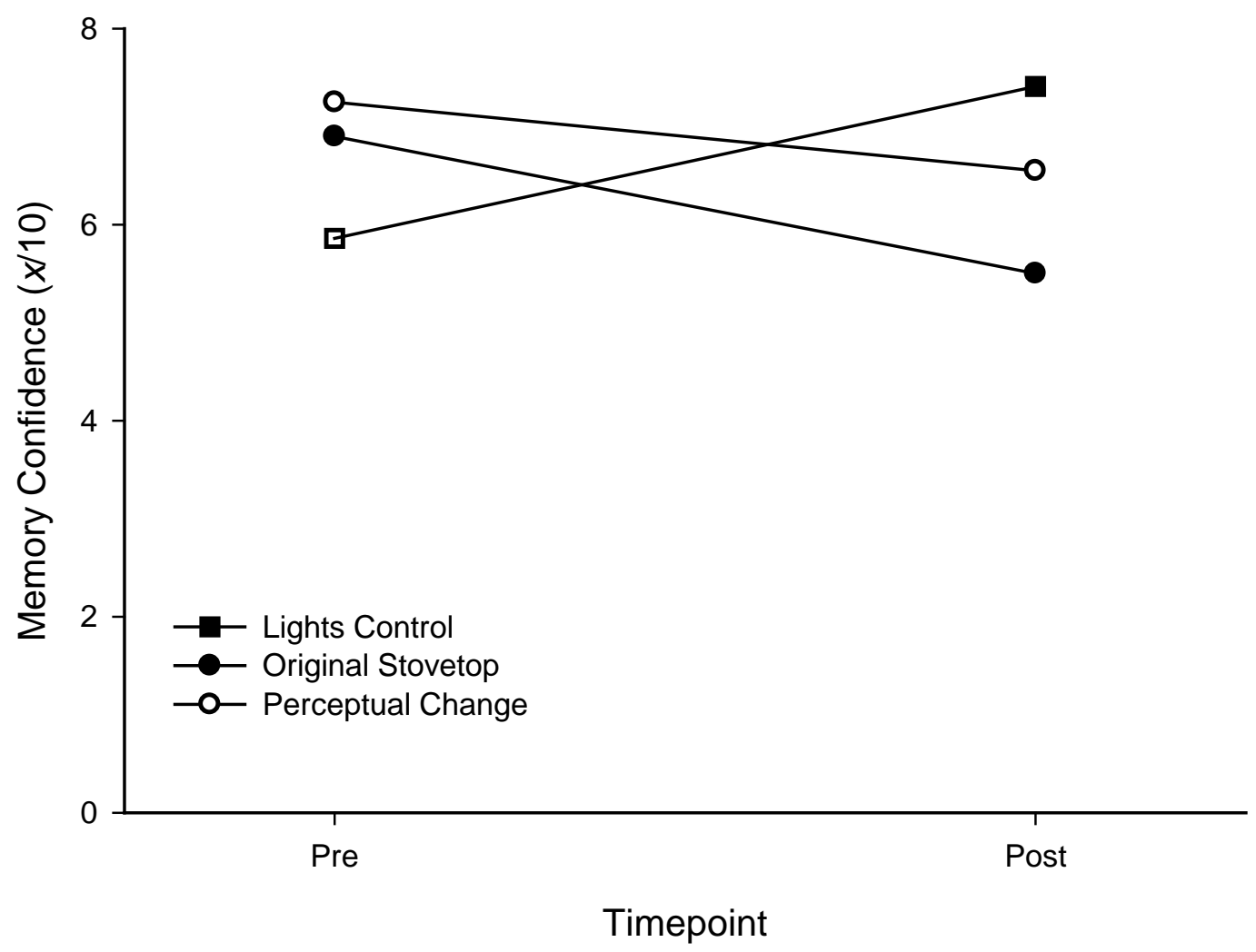

